\title{
Simulation of Contractile Heart Function in the Autodesk Maya Environment Based on Muscle Fiber Macro-Structure
}

\author{
M.V. Titova ${ }^{1}$, T.N. Tomchinskaya ${ }^{1}$ \\ m-titova@list.ru | tomchinskaya@mail.ru \\ ${ }^{l}$ Nizhny Novgorod State Technical University n.a. R.E. Alekseev, Nizhny Novgorod, Russia
}

\begin{abstract}
A dynamic simulation model of the contractile function of the heart is presented. The contractile function simulation is based on the modeling of the muscle fibers' structure according to the Atlas of human anatomy and the use of parameters of their geometric shape as parameters that control the contraction. The basic concepts of the architecture of muscle fibers of the myocardium and the structure of the blood supply to the heart are investigated. An algorithm is developed for local parameterization of the contractile function of the heart, which mimics blood flow and conduction disturbances via special control functions. The algorithm of the simulation model is shown in the example of only the left ventricle of the heart but is embedded in the full three-dimensional model of the ventricular complex of the heart. The simulation model is implemented as a solid-state parameterized model in the Autodesk Maya tool environment, managed by a program in the embedded Python language. The result is compared with the results of the OpenCMISS software in favor of the latter. It is planned to continue work with the implementation of the most advanced concept of the myocardial architecture of TorrentGuasp together with the networks of electrical excitation and blood supply.
\end{abstract}

Keywords: simulation model, human heart, ventricular complex, contractile function.

\section{Introduction}

Despite an unprecedented improvement in computer diagnostics, the doctor still cannot always predict the scenario for the development of the disease of a particular patient. Therefore, the development of patient-oriented interactive mathematical and computer models of organs and systems of the human body remains relevant. This is especially true for the heart and cardiovascular system. In this paper, we discuss the problem of creating a simulation model of the contractile function of the heart, allowing parameterization and, due to this, one or another degree of personalization of the model.

The Maya tool environment was chosen as the modeling environment, which is widely recognized in the field of creating animations, visual effects and games, most of all due to a wide range of features, including individual settings through plug-ins and scripts. Maya contains the built-in scripting programming language MEL (Maya Embedded Language), and from version 8.5 the ability to write scripts in Python has been added. The built-in MEL language is based on $\mathrm{C}++$ and supplemented by the capabilities of the scripting language. Professional $\mathrm{C}++$ developers experience difficulties with MEL due to differences in syntax and lack of debugger for the MEL language.

Using Python in Maya allowed us to lower the threshold for entering scripts and plugins. Python is characterized by higher development speed and ease of maintenance of the code. Existing MEL and C ++ API scripts can be easily ported to Python $[11,20]$, you can use the editor and various Python tools.

The model created in Maya should allow variation by type groups of patients (by age, gender, stage of the disease, and concomitant diseases). Personalization of the model along the way can also solve the problem of detecting and quantifying organ abnormalities according to tomography and, on this basis, automating the diagnosis and monitoring of the patient's condition, including in the early stages of the disease.

\section{Overview of heart patterns}

The work on creating geometric models of the heart has been going on for quite some time. Her first goal was to create threedimensional anatomical atlases. Depending on the purpose of applying the heart model, the composition of its elements, the composition, and complexity of mathematical models, and a set of functions can change. Models for digital medicine require biologically and physiologically correct implementation and visualization.

Physiologically and anatomically correct anisotropic modeling of the contractile function of the heart requires knowledge of the muscle structure and orientation of muscle fibers in all tissues of the heart $[9,10]$. The task is complex and in functional modeling, most often, researchers are limited to modeling only the left ventricle.

If the muscular nature of the heart was established back in $1663[5,6]$, the problem of identifying a unique rule that could be applied equally well to all fibers of the ventricular myocardium, showing their connections in a coordinated, general architectural plan $[1,2]$. This task was seen as the final missing link between the shape of the ventricles and their function.

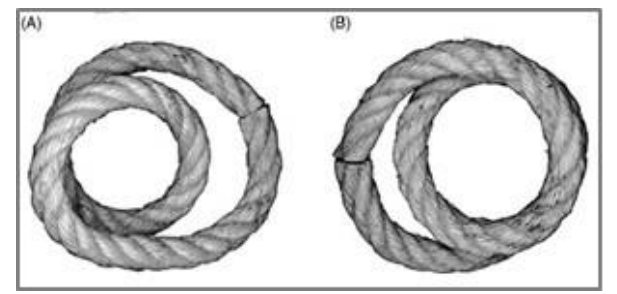

Fig. 1. Two-dimensional spiral model of a rope simulating a fiber path on the base ventricle (A) and its apex (B) [5].

So in [6], it is noted that there is no complete scientific consensus on fundamental issues about the normal and abnormal structure and function of the heart. To solve the problem, the concept of the "Spiral Ventricular Myocardial Tape" by TorrentGuasp (HVMB) was proposed, which is a revolutionary new concept for understanding the global, three-dimensional and functional architecture of the ventricular myocardium (Fig. 1.2).

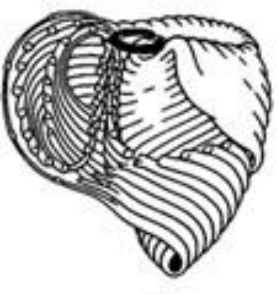

(A)

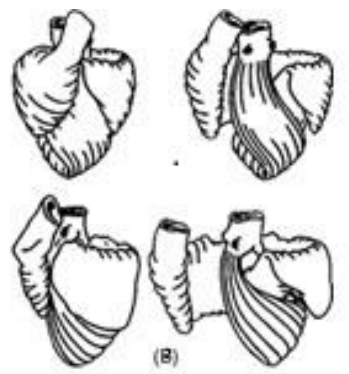

Fig. 2. (A) A drawing made by Torrent-Guasp illustrating the complex three-dimensional fiber architecture of the ventricular body; (B) a schematic representation using a silicone rubber mold (front and left views) [6].

This concept defines the main, cumulative vectors that combine the architecture of the tissue (i.e., shape) and the total 
forces developed (i.e., function) within the body of the ventricles, and will overcome some of the difficulties that modern efforts to create a complex mathematical model of the heart encounter. The concept seems extremely simple in principle, but surprisingly complex in detail $[13,14]$.

It is this concept of myocardial fiber geometry that was taken as the basis by the developers of the Physiome project (http://physiomeproject.org, last updated in 2017).

The Auckland group, examining the anatomical structure of the heart, provided additional evidence that the ventricular myocardium "should not be considered as a uniformly continuous structure" [7,12] (Fig. 3).

In recent years, the task of personalizing the model according to objective medical research, primarily according to tomography [16], has been actively solved.

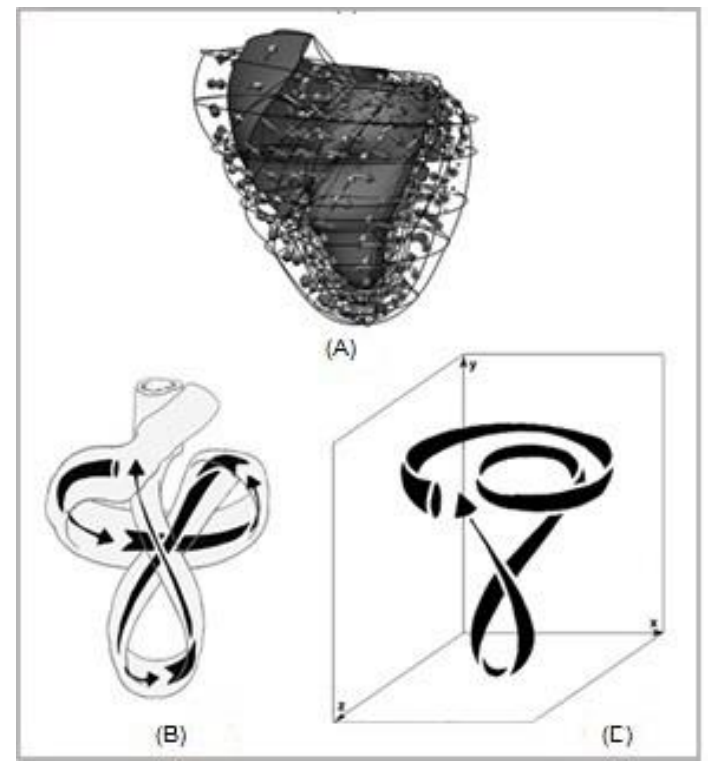

Fig. 3. (A) Many spatially temporarily dependent finite vector forces in the body of the ventricles.

(B and C) the spiral ventricular myocardial tape (HVMB) as a spatial and temporal continuum, combining tissue architecture (i.e. form) and forces developed (i.e. function) inside the ventricular body. (A) (Peter J. Hunter,

University of Auckland, Institute of Bioengineering).

In work [3], a precise quantitative assessment of perfusion and determination of myocardial blood flow was carried out noninvasively using a dynamic image, using also data from positron emission tomography (PET).

A detailed micro- and macro- architecture of the vascular network of the heart (from the epicardial to the capillary (Fig. 4) was revealed. It can be seen from this work that the blood supply system of the heart does not follow the architecture of muscle fibers (see Figs. 1-3).

Three-dimensional models of the heart muscle in modern medicine are actively used in surgery for a preliminary study of the upcoming operation. As a rule, three-dimensional models are built according to the results of computed tomography, and then they are edited and additionally printed [17].

There is a growing interest in dynamic models of electrical and muscle activity of the heart, which require adequate geometric modeling of the heart [23]. A study was conducted in [8], the purpose of which was to test the ability of simulation models of a particular patient to reproduce the response to cardiac resynchronization therapy (CPT) using the latest heart simulation technology.

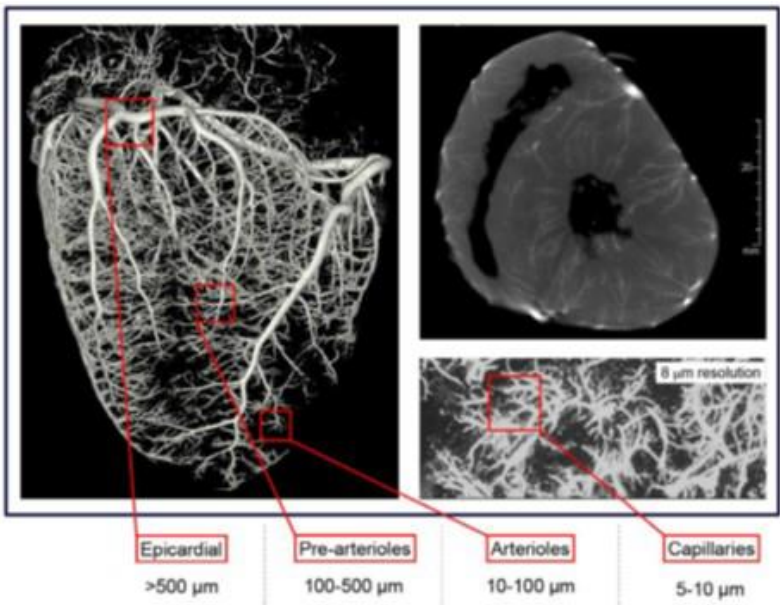

Fig. 4. Functional components of the coronary arterial system.

As a result, it was found that multiscale heart modeling for a particular patient can successfully reproduce the response to CRT. With further testing, this method may be a useful tool in making clinical decisions.

Automation methods for digital heart reconstruction have also begun to be applied. So in [22], based on CT data, the voxel muscle framework of all 4 chambers of the heart is reconstructed, then this framework is smoothed and used to construct the finite element mesh. Such a grid further requires loading data on tissue filling into it, which in turn should be the result of automatic segmentation and classification of the type and condition of tissues according to three-dimensional medical examinations (CT, MRI, ultrasound).

Today, real results have been obtained in the creation of organ tissues using bioprinters, and technologies are known that can be used to create vessels of various diameters. According to authoritative sources in the United States, by 2030, threedimensional organ bioprinting technology will be available to patients [15].

Already, several companies in Germany and the USA are conducting operations with the chondro-spheres and osteospheres (they recreate cartilage and bone tissue). At the same time, chondrocytes are taken from healthy cartilage, grown in the patient's serum, and introduced into the damaged areas.

For the first time in history, scientists at Tel Aviv University printed a heart created using artificially grown human tissues and blood vessels (the size of a rabbit's heart) on a 3D printer [21]. Although previously scientists were already able to depict the structure of the heart using a 3D printer, the complexity of the organ with all the blood vessels, ventricles, and cameras was not represented in the models obtained. And, of course, they were not made from human fabrics.

To date, the creation of geometric models has gained new breath in connection with the demand for dynamic simulators of specific functions of organs and master models for their personalization according to patient data. A rather serious electronic resource of heart models has been accumulated [4]. Among the models of this resource there are both static and dynamic models. But there are no ones who would change their behavior under differentiated external influences.

In particular, there are no dynamic models of muscle activity of the heart that take into account the direction of the fibers of the heart muscle and allow for the control of muscle activity.

The task of creating such a model of the heart is solved in this paper. The result should be a parameterization of heart contractions by changing the parameters of the leading muscle fibers of a complex structure. 


\section{Requirements for the model}

Now there are a large number of software environments for creating 3D models, and not all of them are suitable for the above tasks. Minimum requirements of the solved problem of a general nature: the model must be solid-state highly detailed; parameterized; dynamic; textured. Additional requirements for this research:

1) the anatomical completeness of the model: the presence of all the heart systems necessary for the study: conducting, vascular (as on the Fig.4), muscle (as on the Fig.2 or on the Fig.5);

2) the functional adequacy of systems and controllability of the studied factors [9];

3) functional and topographic interconnectedness of systems.

The integral main requirement for the model is the requirement of anatomical and functional adequacy and the possibility of using the simulation results in clinical practice, both for normal heart activity and for the analysis of pathological processes.

\section{The geometric model of the heart}

For the lack of a more accurate source in our experimental implementation, the structure of the myocardial muscle fiber, is corresponding to the 1980 concept (based on the Atlas of Human Anatomy source, authors: RD Sinelnikov, Ya. R. Sinelnikov, Figure 5), preceding the concept, was taken as the basis TorrentGuasp (Fig. 2) and somewhat simplified in comparison with it.

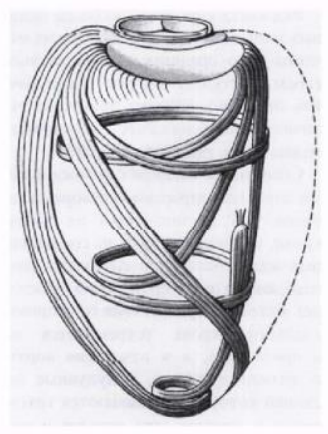

Fig. 5. The direction of muscle bundles of the wall of the left ventricle according to the concept of 1980 (given according to the Atlas of human anatomy).

Simulation of contractions is based on controlling the deformation of the boundaries of each patch of the geometric model (Fig. 6) based on the local value of the angle of inclination of the nearest muscle fiber.

The surfaces bounding the left ventricle are initially defined according to the model built in the thesis of S.F. Pravdina "Mathematical modeling of the structure and function of the left ventricle of the heart" [18]: the external (epi) and internal (endo) surfaces of the LV - as surfaces of revolution in cylindrical coordinates $(\rho, \psi, z)$ :

$$
\begin{aligned}
& \rho_{\text {endo ; epi }}(\psi)=r_{a ; b}(\varepsilon \cdot \cos \psi+(1-\varepsilon)(1-\sin \psi)) ; \\
& \mathrm{z}_{\text {endo; epi }}(\psi)=z_{a ; b}(1-\sin \psi)
\end{aligned}
$$

where $\varepsilon \in[0,1]$ - is a parameter defining the shape of the LV wall from the cone at $\varepsilon=0$, to the ellipsoid of revolution, at

$\varepsilon=1 ; r_{a}, r_{b}$ - are the radii of the inner and outer surfaces at the equator; $\psi$ - latitude (from 0 to 90 ). The parametric mesh created on this surface during the contraction will be deformed by the corresponding control muscle fiber.
The main idea, in comparison with the previous work [19], is to organize in the simulator the ability to control deviations from the norm associated with malnutrition of the heart muscle

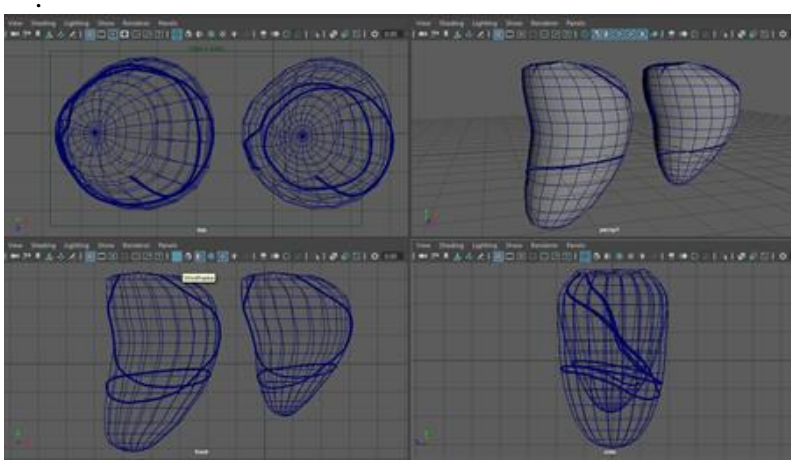

Fig. 6. The outer surface of the left ventricle of the heart, in the conditions of diastole and systole, and a model of one of the muscle fibers that control the contraction.

Such violations can lead to a local weakening of the contractile function of the myocardium, a violation of the temporal relationships between the phases of contraction. Accordingly, the following were introduced: 1) the control function of the phase delay of the process of contraction of the left ventricle (LV), which can be caused by a violation of conduction or a violation of blood supply; 2) the control function of the weakening of the contractile function, depending on the coordinate along the axis of the $\mathrm{LV}$, with the beginning at the lower point of the ventricle (Fig. 7).

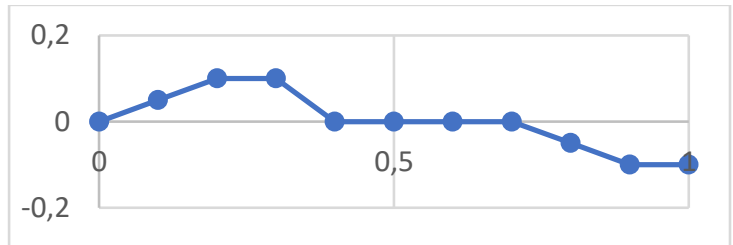

a)

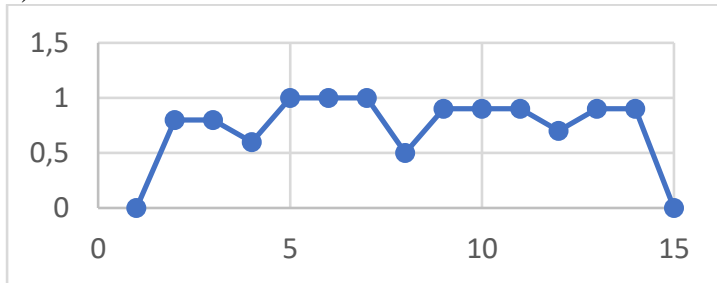

b)

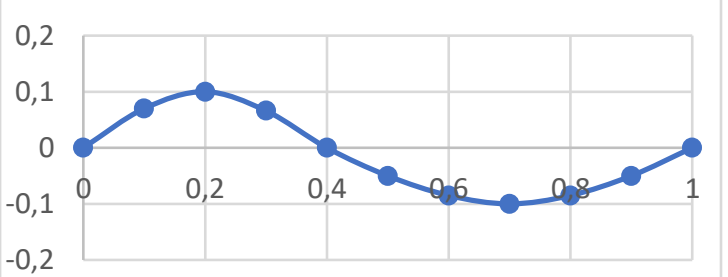

c)

Fig. 7. An example of control schedules: a) the control function of the phase delay of the process of contraction of the left ventricle (LV); b) the function of controlling the weakening of the contractile function, c) the function that controls the simulation of the real rotational dynamics of the heart. 
Additionally, a function of the angular displacement of the heart relative to the vertical axis was introduced, which controls the simulation of the real rotational dynamics of the heart (Fig. 7).

One fiber simulates the entire muscle tape considered in the Torrent-Guasp concept. Currently, the number of fibers is close to the number of LV muscle tapes in the Torrent-Guasp concept.

The reduction starts not from the fibrous ring, as in the previous version [19], but from the lower point of the LV, similar to how it was done in the project at http://opencmiss.org/ (as part of http://physiomeproject.org , and http://www.torrentguasp.com/), where the bottom point is considered to be fixed. From layer to layer, the following procedure is performed: the lower border of the patch remains unchanged, and the upper and side borders of the current patch change in the proportion specified by the angular position of the muscle fiber that controls the patch.

The whole process is controlled by a Python program executed by system tools of the Autodesk Maya environment (Fig. 8).

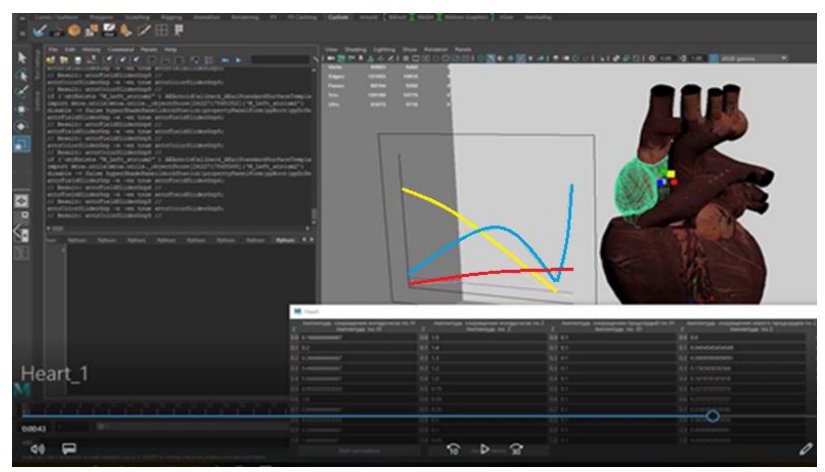

Fig. 8. The final heart model, control functions and tables to control the simulation of its contractions in Autodesk Maya.

\section{Conclusion}

The model that we constructed and the simulator of the contractile function of the heart in the experiment demonstrate the control possibilities laid down and discussed above. To evaluate the result, a comparison was made with the model of the ventricular complex on the website http://opencmiss.org/, built by the developers of the Physiome project based on the concept of the Torrent-Guasp architecture. It turned out that the complexity and physiology of the movement that our model demonstrates are inferior to the model on the website http://opencmiss.org/. At the same time, the analysis shows that in both models the requirement of the functional and topographic interconnection of the muscular, circulatory and conduction systems of the heart is not fully or partially fulfilled. In particular, they lack control associated with impaired conduction of electrical excitation in the heart: in the atrial zone, atrioventricular node, and ventricular zone. It is planned to develop the model accordingly to the ability to take into account local disturbances in blood supply and related disturbances in the conductivity of electrical excitation and disturbances in the nutrition of the heart muscle. The solution to this problem is complicated both by the fact that the micro- and macroarchitecture of the vascular network does not coincide with the architecture of muscle fibers and by the fact that for the simulation it will also be necessary to update the model with the data of the patient's medical studies.

\section{References}

[1] Buckberg GD,Weisfeldt ML, Ballester M, Beyar R, Burkhoff D, Coghlan HC, Doyle M, Epstein ND, Gharib M, Ideker RE, Ingels NB, LeWinter MM, McCulloch AD, Pohost GM, Reinlib RJ, Sahn DJ, Spinale FG, Spotnitz HM, Sopko G, Torrent-Guasp F, Shapiro EP. Left ventricular form and function: scientific priorities and strategic planning for development of new views of disease. Circulation 2004;110:e333-6.

[2] Buckberg GD. Architecture must document functional evidence to explain the living rhythm. Eur J Cardiothoracic Surg 2005;27:202-9.

[3] Feher A., Sinusas A.J., Quantitative Assessment of Coronary Microvascular Function // Circ. Cardiovasc. Imaging. $2017.21 \mathrm{p} . \quad$ DOI: 10.1161/CIRCIMAGING.117.006427.

[4] Human Heart 3D Models [Электронный pecypc] https://www.turbosquid.com/3d-model/human-heart . (Дата обращения: 08.08.2019).

[5] Kardel T. Steno on muscles: introduction, texts, translations // Trans Am Phylos Soc 1994;84(1):58-75.

[6] Kocica M.J., Corno A.F., Carreras-Costa F., BallesterRodes M., Moghbel M.C., Cueva C.N.C., Lackovic V., Kanjuh V.I., Torrent-Guasp F. The helical ventricular myocardial band band: global, three-dimensional, functional architecture of the ventricular myocardium (Review)// European.

[7] LeGrice IJ, Takayama Y, Covell JW. Transverse shear along myocardial cleavage planes provides a mechanism for normal systolic wall thickening. Circ Res 1995;77:182 — 93.

[8] Okada J. et al. Multi-scale, tailor-made heart simulation can predict the effect of cardiac resynchronization therapy // Journal of Molecular and Cellular Cardiology. V.108, July 2017, P. 17-23.

[9] Pravdin S.F., Berdyshev V.I., Panfilov A.V., Katsnelson L.B., Solovyova O., Markhasin V.S.. Mathematical model of the anatomy and fibre orientation field of the left ventricle of the heart // Biomedical Engineering Online, 12:54, 2013. $21 \mathrm{p}$.

[10] Pravdin S.F., Dierckx H., Katsnelson L.B., Solovyova O., Markhasin V.S., Panfilov A.V.. Electrical wave propagation in an anisotropic model of the left ventricle based on analytical description of cardiac architecture // PLOS One. 2014. PLoS ONE 9(5): e93617.

[11] Robert Galanakis, Practical Maya Programming with Python / Published by Packt Publishing Ltd., 2014, 354p., ISBN 978-1-84969-472-8.

[12] Smaill BH, LeGrice IJ, Hooks DA, Pullan AJ, Caldwell BJ, Hunter PJ. Cardiac structure and electrical activation: models and measurement. Proc Austral Physiol Pharm Soc 2004;34:141-9.

[13] Torrent-Guasp F, Kocica MJ, Corno A, Komeda M, Cox J, Flotats A, Ballester-Rodes M, Carreras-Costa F. Systolic ventricular filling. Eur J Cardiothorac Surg 2004; 25(3): $376-86$.

[14] Torrent-Guasp F, Kocica MJ, Corno AF, Komeda M, Carreras-Costa F, Flotats A, Cosin-Aguillar J, Wen H. Towards new understanding of the heart structure and function. Eur J Cardiothorac Surg 2005;27:191—201.

[15] Renowned scientist Vladimir Mironov, tissue engineering, the author of the press technology in Moscow https://www.mirprognozov.ru/prognosis/science/izvestnyiy -uchenyiy-vladimir-aleksandrovich-mironov/ (24.08.2019).

[16] Matveyenko V.P., Shardakov I.N., Shestakov A.P. Algorithm for creating three-dimensional images of human organs using tomography data// ISSN 1812-5123 Russian Journal of Biomechanics. 2011. Vol. 15, No. 4 (54): 15$27 \mathrm{pp}$. 
[17] Operatsyi s ispolzovaniem modeley serdsa otpechatannyh na 3D printere. [Electronic resource, in Russian] - URL: www.printfuture.ru /2017/ (25.08.2019).

[18] Pravdin S.F. Mathematical modeling of the structure and function of the left ventricle of the heart / Synopsis of the dissertation for the degree of candidate of physical and mathematical sciences, 2015. 20p (in Russian).

[19] Titova M.V., Tomchinskaya T.N. Development of a simulation model of the contractile function of the heart in Autodesk Maya // GraphiCon 2018: Proceedings of the 28th International conf. Computer Graphics and Machine Vision. Tomsk, Sept. 24-27, 2018. - 511 p. ISSN 2618-8317 (in Russian).

[20] Wilkins M.R., Kazmier K. MEL Scripting for Maya Animators. -2nd Edition, 2005, -548 pp.

[21] Tel Aviv University Scientists Print First 3D Heart Using Patient's Own Cells. www.breakingisraelnews.com/126504/first-3d-heartusing-patients-cells/ (24.08.2019).

[22] Shardakov I.N., Shestakov A.P. Construction of the 4chamber geometrical image of human heart based on x-ray tomography // Russian Journal of Biomechanics. 2015. V. 19, No 4: 320-331. DOI: 10.15593/RJBiomech/2015.4.04.

[23] Shestakov A.P./ Mathematical modeling of myocardial electrodynamics and analysis of factors affecting its modes. - Dissertation for the degree of candidate of physical and mathematical sciences, Inst.MSS UrO RAN, 2019. -117p (in Russian). 